\title{
Parameter space and model reduction with shape parametrization, by means of active subspaces and POD-Galerkin methods for industrial and biomedical applications
}

Marco Tezzele, Francesco Ballarin, Andrea Mola and Gianluigi Rozza

Mathematics area, mathLab, SISSA, International School for Advanced Studies, Trieste, Italy

\begin{tabular}{|l|}
\hline Introduction \\
\hline We introduce a new framework for parameters space reduction in naval and biomedical \\
engineering obtained by coupling: Active Subspaces property to identify lower dimensional \\
structure in the parameters space [1]; Free Form Deformation (FFD) and Radial Basis \\
Functions (RBF), to morph the geometry; Response surfaces method (RS) and POD- \\
Galerkin methods. \\
$\qquad \begin{array}{l}\text { RBD } \\
\text { Active Subspaces }\end{array}$
\end{tabular}

The Active Subspaces Property

Consider a function, its gradient vector and a sampling density

$$
f=f(\mathbf{x}), \quad \mathbf{x} \in \mathbb{R}^{m}, \quad \nabla f(\mathbf{x}) \in \mathbb{R}^{m}, \quad \rho: \mathbb{R}^{m} \rightarrow \mathbb{R}_{+}
$$

Take the average outer product of the gradient and partition its eigendecomposition,

$$
\mathbf{C}=\mathbb{E}\left[\nabla_{\mathbf{x}} f \nabla_{\mathbf{x}} f^{T}\right]=\int\left(\nabla_{\mathbf{x}} f\right)\left(\nabla_{\mathbf{x}} f\right)^{T} \rho d \mathbf{x}=\mathbf{W} \boldsymbol{\Lambda} \mathbf{W}^{T}
$$$$
\boldsymbol{\Lambda}=\left[\begin{array}{ll}
\boldsymbol{\Lambda}_{1} & \\
& \boldsymbol{\Lambda}_{2}
\end{array}\right], \quad \mathbf{W}=\left[\begin{array}{ll}
\mathbf{W}_{1} & \mathbf{W}_{2}
\end{array}\right], \quad \mathbf{W}_{1} \in \mathbb{R}^{m \times n}
$$

Rotate and separate the coordinates: $\mathbf{x}=\mathbf{W W}^{T} \mathbf{x}=\mathbf{W}_{1} \mathbf{W}_{1}^{T} \mathbf{x}+\mathbf{W}_{2} \mathbf{W}_{2}^{T} \mathbf{x}=\mathbf{W}_{1} \mathbf{y}+\mathbf{W}_{2} \mathbf{z}$ We have that $\mathbf{y}=\mathbf{W}_{1}^{T} \mathbf{x} \in \mathbb{R}^{n}$ is the active variable and $\mathbf{z}=\mathbf{W}_{2}^{T} \mathbf{x} \in \mathbb{R}^{m-n}$ the inactive one.
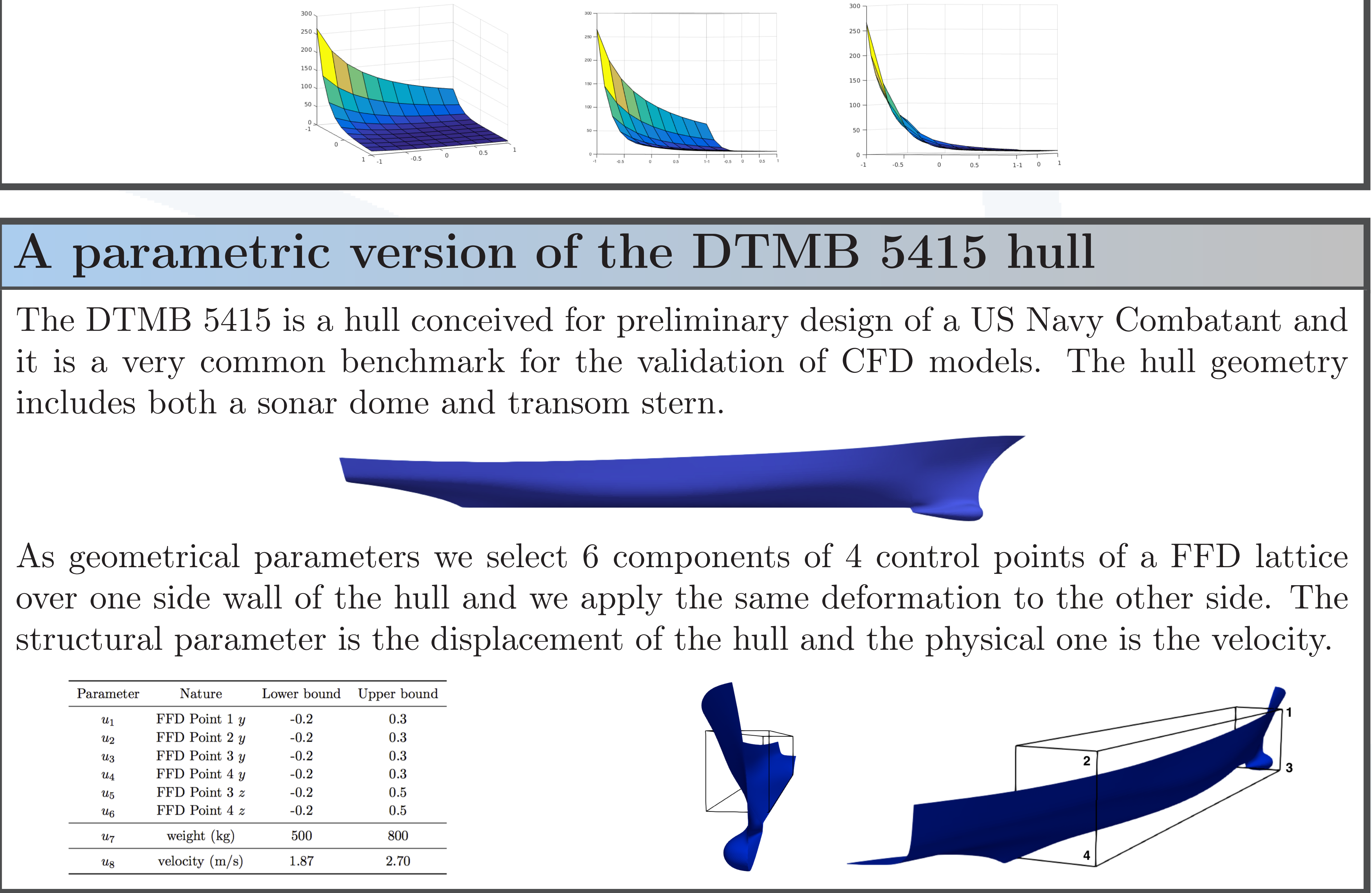

Eigenvalues and error analysis

We approximate the gradients of the wave resistance with respect to the parameters and look for a spectral gap of the $\mathbf{C}$ matrix.

We underline the presence of a major gap between the first and the second eigenvalue and a minor one between the second and the third.

The sufficient summary plot $\left(f(\mathbf{x})\right.$ against $\left.\mathbf{W}_{1}^{T} \mathbf{x}\right)$ confirms the presence of an active subspace of dimension 1 and 2 .
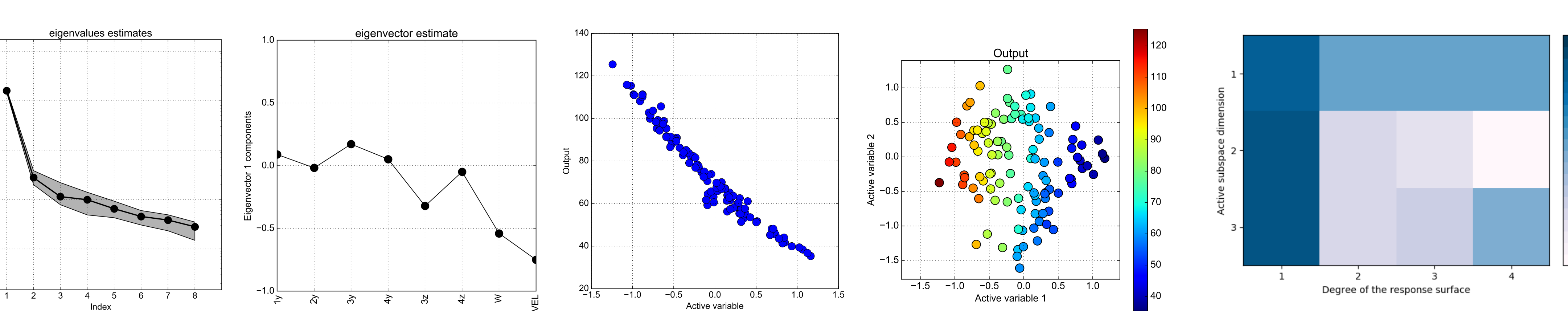

Using a response surface of order 4 and an active subspace of dimension 2 ensures an error on the test dataset equal to $2.5 \%$. It is a good starting point to perform further optimization in the ridge approximation context. Doing so we reduced the parameter space from dimension 8 to 2 .

\begin{tabular}{l}
\hline Geometrical Deformation: the PyGeM library \\
\hline PyGeM is a python library using Free Form Deformation, Inverse Distance \\
Weighting, and Radial Basis Function interpolation to parametrize and \\
morph complex geometries. \\
It interacts with industrial file formats used for CAD management (.iges, \\
.step, .stl), mesh files (.unv and OpenFOAM), and output files (.vtk). \\
See github.com/mathLab/PyGeM and mathlab.sissa.it/cse-software
\end{tabular}

\section{Carotid parametrization}

Vessels geometry strongly influences hemodynamics behaviour. We study the influence of the vessel shape on blood flow.

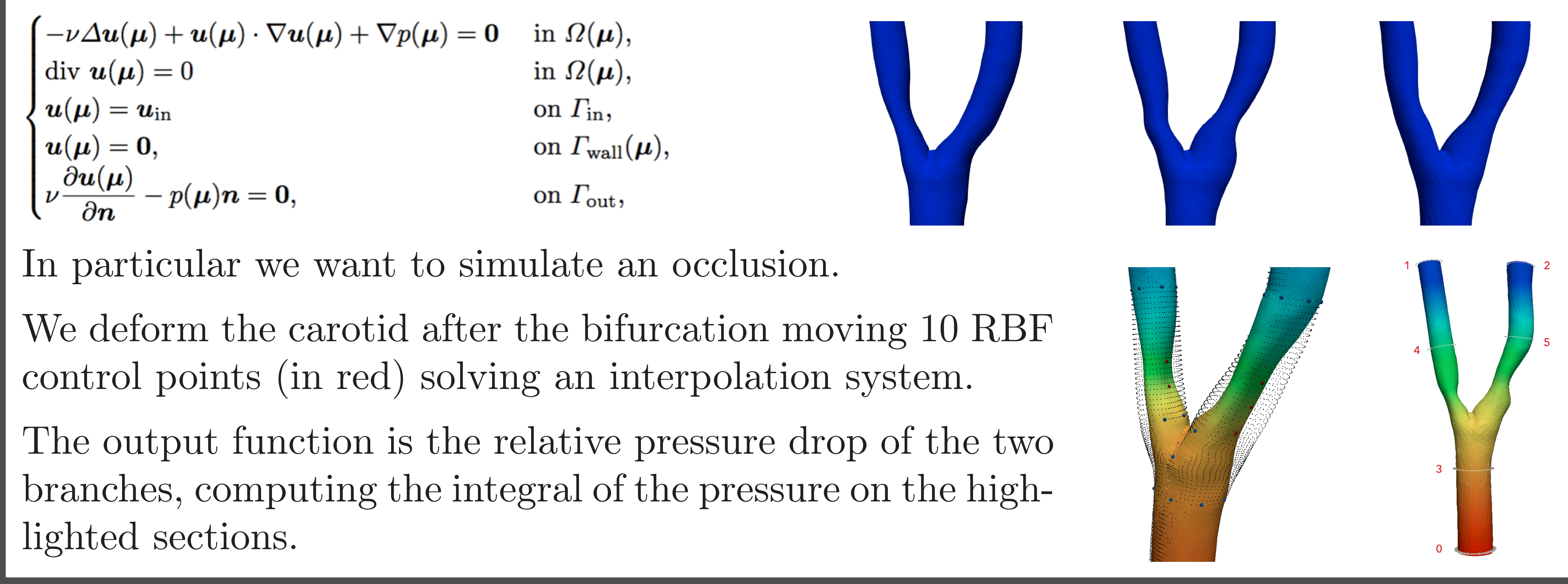

Spectral and POD analysis

The presence of an active subspace of dimension one is clear both from the spectral analysis and the sufficient summary plot.

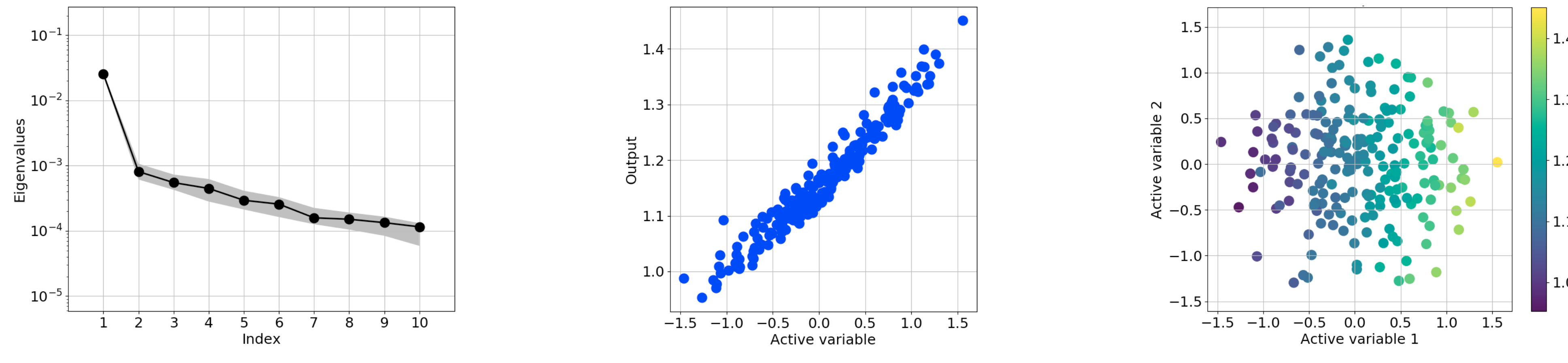

The two dimensional active subspace spanned by the first two eigenvectors of the covariance matrix seems to better capture the behaviour of the output function. We use this information perform a further reduction by a POD-Galerkin ROM.

We exploit a 2-dimensional active subspace to compute the POD snapshots in a reduced space with respect to the full 10-dimensional parameter space.

Typical reduced space dimensions and computational speedup for cardiovascular flows. In particular the speedup from high-fidelity simulations to reduced-order ones: 500:1.

Here the POD singular values for velocity, supremizers and pressure, as a function of the number $\mathrm{N}$ of selected POD modes:

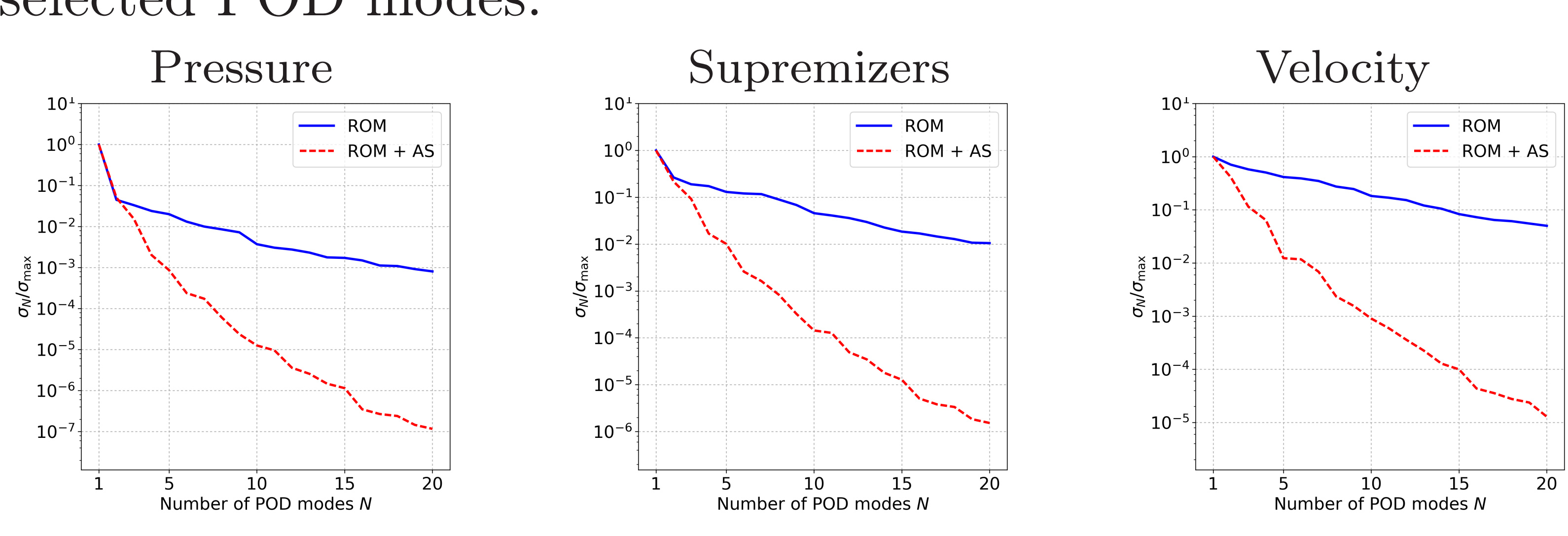

The results show a slower decay for the standard approach when compared to the combined one, meaning that the standard approach has to deal with a considerably larger solution manifold

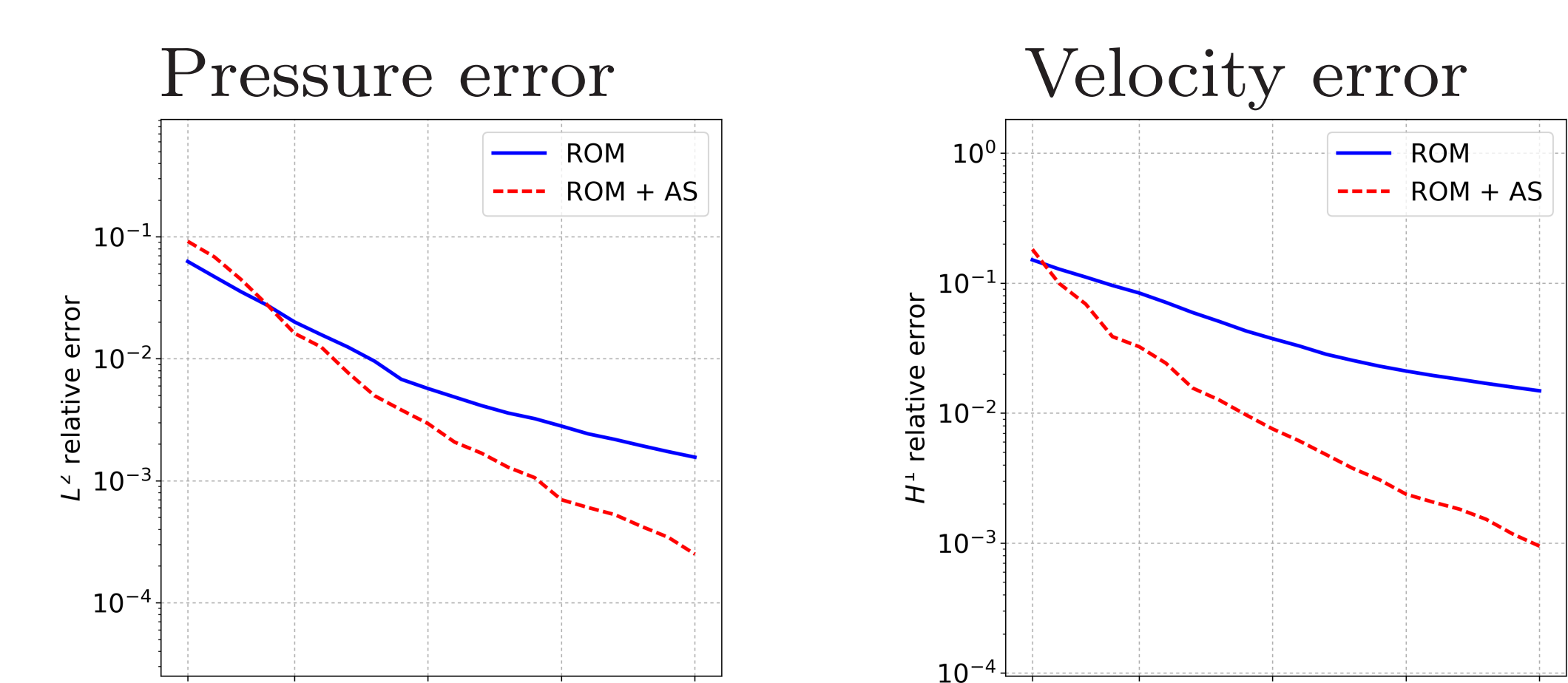

The combined methodology is able to reach relative errors which are up to an order of magnitude smaller when compared to the standard one, for both velocity and pressure when $N=20$.

\section{References}

[1] P. G. Constantine. Active subspaces: Emerging ideas for dimension reduction in parameter studies, volume 2. SIAM, 2015.

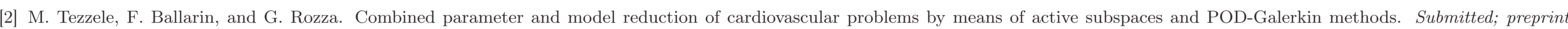
arXiv:1711.10884, 2017

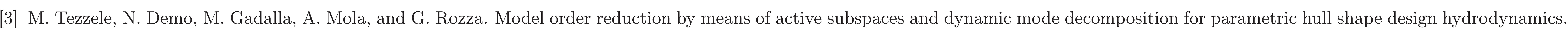
Submitted; preprint arXiv:1803.07377, 2018.

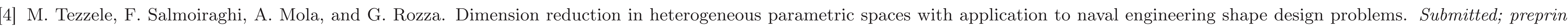
arXiv:1709.03298, 2017 